\title{
The M1 form of tumor-associated macrophages in non-small cell lung cancer is positively associated with survival time
}

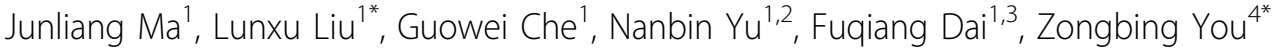

\begin{abstract}
Background: Tumor-associated macrophages (TAMs) play an important role in growth, progression and metastasis of tumors. In non-small cell lung cancer (NSCLC), TAMs' anti-tumor or pro-tumor role is not determined. Macrophages are polarized into M1 (with anti-tumor function) and M2 (with pro-tumor function) forms. This study was conducted to determine whether the M1 and M2 macrophage densities in NSCLC are associated with patient's survival time.

Methods: Fifty patients with an average of 1-year survival (short survival group) and 50 patients with an average of 5-year survival (long survival group) were included in this retrospective study. Paraffin-embedded NSCLC specimens and their clinicopathological data including up to 8-year follow-up information were used. Immunohistochemical double-staining of CD68/HLA-DR (markers for M1 macrophages) and CD68/CD163 (markers for M2 macrophages) was performed and evaluated in a blinded fashion. The M1 and M2 macrophage densities in the tumor islets, stroma, or islets and stroma were determined using computer-aided microscopy. Correlation of the macrophage densities and patient's survival time was analyzed using the Statistical Package for the Social Sciences.

Results: Approximately 70\% of TAMs were M2 macrophages and the remaining 30\% were M1 macrophages in NSCLC. The M2 macrophage densities (approximately 78 to 113 per $\mathrm{mm}^{2}$ ) in the tumor islets, stroma, or islets and stroma were not significantly different between the long survival and short survival groups. The M1 macrophage densities in the tumor islets (approximately $70 / \mathrm{mm}^{2}$ ) and stroma (approximately $34 / \mathrm{mm}^{2}$ ) of the long survival group were significantly higher than the $\mathrm{M} 1$ macrophage densities in the tumor islets (approximately $7 / \mathrm{mm}^{2}$ ) and stroma $\left(13 / \mathrm{mm}^{2}\right)$ of the short survival group $(P<0.001$ and $P<0.05$, respectively). The $M 2$ macrophage densities were not associated with patient's survival time. The M1 macrophage densities in the tumor islets, stroma, or islets and stroma were positively associated with patient's survival time in a univariate analysis $(P<0.01$ or 0.001$)$. In a multivariate Cox proportional hazards analysis, the M1 macrophage density in the tumor islets was an independent predictor of patient's survival time.
\end{abstract}

Conclusions: The M1 macrophage density in the tumor islets is an independent predictor of survival time in NSCLC patients.

\footnotetext{
* Correspondence: lunxu_liu@yahoo.com.cn; zyou@tulane.edu

'Department of Thoracic and Cardiovascular Surgery, West China Hospital,

Sichuan University, Chengdu 610041, China

${ }^{4}$ Departments of Structural \& Cellular Biology and Orthopaedic Surgery,

Tulane Cancer Center, LCRC, Tulane Center for Aging, Tulane University

School of Medicine, New Orleans, LA 70112, USA
} 


\section{Background}

Non-small cell lung cancer (NSCLC) remains the most common cause of cancer-related death worldwide. Metastasis may have occurred at the time of initial diagnosis, even at a very early stage such as stage IA. That explains why the five-year survival rate is approximately $67 \%$ for patients with stage IA NSCLC after putatively curative surgical resection [1]. Tumor cells use multiple mechanisms to invade extracellular matrix and metastasize to distant organs. The interaction between the tumor cells and stromal cells in the tumor microenvironment plays an important role in tumor growth and metastasis. Macrophages are prominent stromal cells in this interaction. They secret a variety of growth factors, cytokines, chemokines, and enzymes that regulate tumor growth, angiogenesis, invasion, and metastasis [2].

Recently, it has been well recognized that tumor-associated macrophages (TAMs) are not homogenous [3]. Microlocalization, in terms of where macrophages are observed under a microscope, is an important factor for prognosis. Increased number of macrophages within the tumor islets confers a marked survival advantage, whereas increased number of macrophages in the tumor stroma is associated with poor prognosis in NSCLC [4]. In addition, macrophages are polarized into two functionally distinct forms M1 and M2, mirroring the Th1 and Th2 nomenclature of T cells [3]. Differentiation of the M1 macrophages is induced by interferon- $\gamma$, lipopolysaccharides, tumor necrosis factor (TNF) $\alpha$, and granulocyte-monocyte colony-stimulating factor. The M1 macrophages produce high levels of interleukin (IL)-12, IL-23, TNFa, IL-1, IL-6, CXC ligand 10 (CXCL10), inducible nitric oxide synthase (iNOS), human leukocyte antigen (HLA)-DR, and reactive oxygen and nitrogen intermediates [3,5-7]. Differentiation of the M2 macrophages is induced by IL-4, IL-10, IL-13, IL-21, activin A, immune complexes, and glucocorticoid [3]. The M2 macrophages express high levels of IL-10, IL1 receptor antagonist, CC ligand 22 (CCL22), scavenger, mannose receptor, galactose receptor, arginase I, and CD163 antigen $[3,8]$. Ohri et al recently reported that the M1 macrophage density in the tumor islets is positively associated with extended survival of NSCLC patients [9].

We have previously reported that the number of TAMs in the tumor islets and the ratio of TAMs in the tumor islets versus stroma are positively associated with survival time in patients with NSCLC [10]. In this study, we further determined that the M1 form of TAMs is an independent prognostic factor in patients with NSCLC.

\section{Methods}

\section{Study population}

This study was approved by the Institutional Review Board of West China Hospital, Sichuan University. The procedures to obtain human lung cancer tissues and follow-up information are in accordance with the Ethical Principles for Medical Research Involving Human Subjects as formulated in the World Medical Association Declaration of Helsinki (revised in 2008). All specimens were obtained from the archives of formalin-fixed, paraffin-embedded tissue blocks in the Department of Thoracic and Cardiovascular Surgery, West China Hospital, Sichuan University. The lung cancer tissues were collected from surgeries performed from June, 1999 to August, 2001. The patients were followed up until December, 2007, through outpatient visits and/or correspondences to family members. Fifty patients with long survival time (1972.0 \pm 78.0 days) and 50 patients with short survival time (351.8 \pm 32.5 days) were included in this retrospective study. The inclusion criteria were: (1) follow-up data were complete; and (2) paraffin blocks were available; and (3) without pre-operative chemotherapy or radiotherapy. All of the cases that satisfied the inclusion criteria were included in this study. Histological evaluation was based on the World Health Organization criteria. Tumor stage was evaluated according to the International Union against Cancer TNM classification system. The clinicopathological characteristics were summarized in Table 1.

\section{Immunohistochemistry}

Four- $\mu \mathrm{m}$ thick tissue sections were de-waxed in xylene and rehydrated through graded alcohols. Antigen retrieval was carried out using microwave at middle-to-high temperature for $8 \mathrm{~min}$, low-to-high temperature for 5 min, and then cooled down at room temperature for 20 min. Mouse anti-human CD68 monoclonal antibodies (clone KP1, recognizing macrophages), mouse antiHLA-DR monoclonal antibodies (clone LN3, recognizing M1 macrophages), and mouse anti-human CD163 antigen monoclonal antibodies (clone 10D6, recognizing M2 macrophages) were produced by Invitrogen, Carlsbad, $\mathrm{CA}$, and were obtained from Zhongshan Goldenbridge Biotechnology Co., LTD., Beijing, China. Mouse IgG1 (clone NCG01, Abcam, Cambridge, MA, USA) was used as an isotype negative control.

Immunohistochemical staining of individual marker or double-staining of CD68 and CD163 or CD68 and HLA-DR was performed using the DouSP ${ }^{\mathrm{mm}}$ double-stain kit (Maxim-Bio, Fuzhou City, Fujian Province, China) according to the manufacturer's instructions. Development of red color was performed using streptavidin-peroxidase conjugate and 3-Amino-9-ethylcarbazole (AEC). Development of black-purple color was performed using streptavidin-alkaline-phosphatase conjugate and 5bromo-4-chloro-3-indolyl phosphate (BCIP)/nitro blue tetrazolium (NBT). Sections were then counterstained 
Table 1 Clinicopathological characteristics of patients with non-small cell lung cancer $(\mathbf{n}=100)$

\begin{tabular}{|c|c|c|}
\hline Variable & Long survival $(n=50)$ & Short survival $(n=50)$ \\
\hline Survival (days, mean \pm standard error) & $1972.0 \pm 78.0$ & $351.8 \pm 32.5$ \\
\hline Age (years, mean \pm standard error) & $58.0 \pm 1.4$ & $60.5 \pm 1.3$ \\
\hline Gender (male: female) & $40: 10$ & $41: 9$ \\
\hline \multicolumn{3}{|l|}{ Tumor stage: number (\%) } \\
\hline 1 & $24(48)$ & $11(22)$ \\
\hline$\|$ & $9(18)$ & $11(22)$ \\
\hline III & $15(30)$ & $20(40)$ \\
\hline IV & $2(4)$ & $8(16)$ \\
\hline \multicolumn{3}{|l|}{ Histology: number (\%) } \\
\hline Adenocarcinoma & $12(24)$ & $24(48)$ \\
\hline Squamous & $34(68)$ & $17(34)$ \\
\hline Alveolar cell & $3(6)$ & $7(14)$ \\
\hline Large cell & $1(2)$ & $2(4)$ \\
\hline \multicolumn{3}{|l|}{ Tumor grade: number (\%) } \\
\hline Well differentiation & $2(4)$ & $0(0)$ \\
\hline Moderate differentiation & $27(54)$ & $21(42)$ \\
\hline Poor differentiation & $15(30)$ & $15(30)$ \\
\hline Not recorded & $6(12)$ & $14(28)$ \\
\hline \multicolumn{3}{|l|}{ Lymph node metastasis: number (\%) } \\
\hline No & $37(74)$ & $23(46)$ \\
\hline Yes & $13(26)$ & $27(54)$ \\
\hline
\end{tabular}

with hematoxylin and mounted in an aqueous mounting medium. Tissue sections with macrophages previously stained positively were used as positive control, while tissue sections with primary antibodies replaced by mouse isotype IgG1 served as negative control. Five representative high-power fields ( $\times 400$ magnification) per tissue section were selected using an Olympus BX51 microscope (Olympus, Tokyo, Japan). Areas of the tumor islets, tumor stroma, or combination of the tumor islets and stroma were defined and measured using the Image-Pro Plus 6.0 software (Media Cybernetics, Silver Spring, MD, USA). The number of nucleated cells with positive staining for the phenotypic marker in each area was counted. Macrophage density was calculated as cell number per $\mathrm{mm}^{2}$ of the tumor islets, stroma, or islets and stroma. Evaluation of the stained tissue sections was performed by two investigators who were blinded in regard to which group the specimens belonged to. Macrophage density of each case was an average of the results obtained by the two examiners.

\section{Statistical analysis}

Statistical analysis was carried out using the Statistical Package for the Social Sciences (SPSS, version 13.0, SPSS Inc., Chicago, IL, USA). For categorical analysis, the median value of macrophage density was used as a cut-off point to dichotomize the continuous variables. The Mann-Whitney nonparametric test was used to compare between two groups. The Spearman's rank correlation coefficient was calculated to assess any potential relationship between macrophage density and patient's survival time. The Kaplan-Meier survival curves were used to look for correlation between macrophage density and patient's survival time. Statistical significance was analyzed using the log-rank test. A multivariate Cox proportional hazards model was used to estimate adjusted hazard ratios and 95\% confidence intervals (CI) and to identify which form of macrophages was an independent prognostic factor. The validity of the proportional hazards assumption was assessed from log (-log [Survival]) curves. For the above comparisons, $\mathrm{P}<0.05$ was considered statistically significant.

\section{Results}

\section{Patient characteristics}

Among the 100 patients (Table 1) who had undergone surgery, 10 patients received additional chemotherapy after surgery. No patients received radiotherapy before or after surgery. All of the patients had complete follow-up information and the pathological diagnosis was verified by a pathologist prior to inclusion in this study. The overall cumulative survival rates were $68 \%$ for 1 year, $46 \%$ for 3 years, and $32 \%$ for 5 years. The group of patients with long survival time had survived 5.4 years on the average, whereas the group of patients with short survival time had lived only about one year (Table 1). 


\section{Immunohistochemical detection of macrophages}

In order to assess whether the markers chosen actually detect different cellular subsets of macrophages, tissue sections from five patients were initially stained for CD68, CD163, or HLA-DR, alone or in combination. It was found that CD68 staining identified cells with morphological features of macrophages. Among the CD68+ macrophages, some of them stained positively for HLADR, a marker of the M1 macrophages (Figure 1a), whereas others stained positively for CD163, a marker of the M2 macrophages (Figure 1b). In double-staining for CD163 and HLA-DR, a majority of macrophages stained positively for either CD163 or HLA-DR (Figure 1c). Only a small percentage of macrophages (median $3.1 \%$, range $1.2 \%-8.1 \%$ ) stained positively for both CD163 and HLA-DR.

\section{M1 and M2 macrophage densities in the tumor islets and} stroma

When the M1 and M2 macrophages on the tumor sections (including the tumor islets and stroma) were counted under high-power fields, approximately $70 \%$ of macrophages were CD68+/CD163+ M2 macrophages and the remaining $30 \%$ of them were CD68+/HLA-DR+ M1 macrophages. The M1 macrophage density (approximately $70 / \mathrm{mm}^{2}$ ) in the tumor islets and stroma of patients with long survival time was significantly higher (about 4 times) than the M1 macrophage density (approximately $17 / \mathrm{mm}^{2}$ ) in the tumor islets and stroma of patients with short survival time $(\mathrm{P}<0.001)$ (Table 2). The M2 macrophage density in the tumor islets and stroma of the long survival group (approximately $98 / \mathrm{mm}^{2}$ ) was not significantly different from the M2 macrophage density in the tumor islets and stroma of the short survival group (approximately $110 / \mathrm{mm}^{2}$ ) $(\mathrm{P}>0.05)$. The $\mathrm{M} 1 / \mathrm{M} 2$ ratio in the tumor islets and stroma was 0.7 in patients with long survival time and 0.2 in patients with short survival time (Table 2).

When the M1 and M2 macrophages were assessed in the tumor islets or stroma individually, the M1 macrophage densities in the tumor islets (approximately $70 / \mathrm{mm}^{2}$ ) and stroma (approximately $34 / \mathrm{mm}^{2}$ ) of the long survival group were significantly higher than the M1 macrophage densities in the tumor islets (approximately $7 / \mathrm{mm}^{2}$ ) and stroma
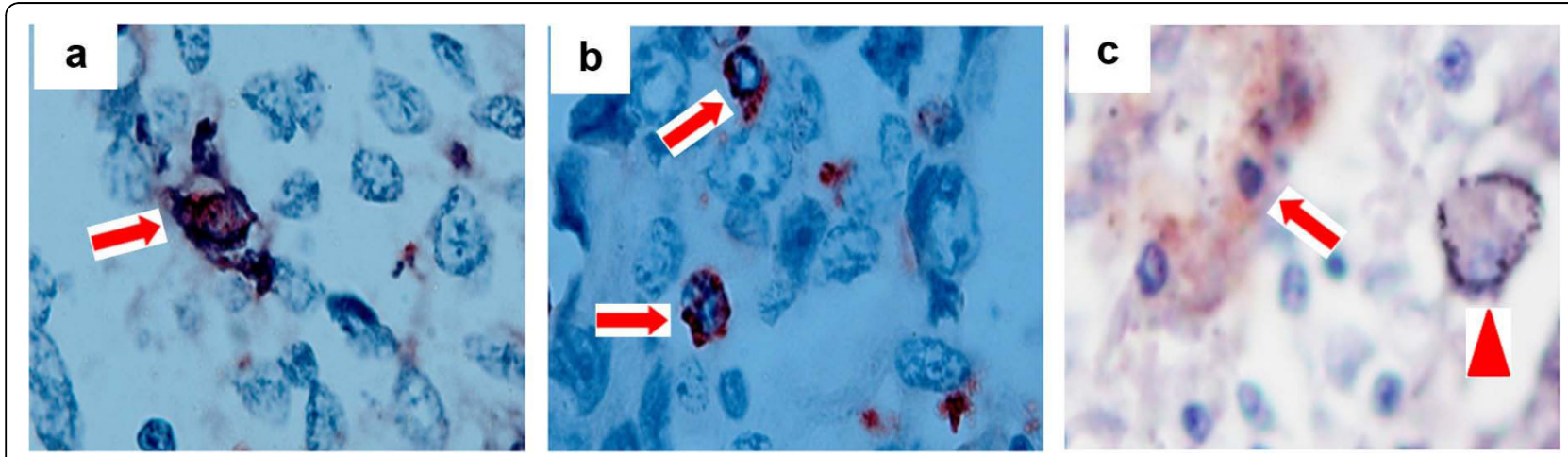

Figure 1 Immunohistochemical detection of macrophages in NSCLC tumor islets. a) The M1 macrophage double stained with the antiCD68 (red) and anti-HLA-DR (black-purple) antibodies (arrow). b) The M2 macrophages double stained with the anti-CD68 (red) and anti-CD163 (black-purple) antibodies (arrow). c) The M1 macrophage marker HLA-DR stained black-purple (arrowhead) and the M2 macrophage marker CD163 stained red (arrow). Original magnification, $\times 1000$ for a $\&$ b, and $\times 400$ for $c$.

Table 2 Density and microlocalization of macrophages in non-small cell lung cancer

\begin{tabular}{llllllll}
\hline & \multicolumn{3}{c}{ Long survival } & \multicolumn{3}{c}{ Short survival } & \multicolumn{1}{c}{ I +S] long/ } \\
\cline { 2 - 7 } M Form & Islets & Stroma & $\mathbf{I}+\mathbf{S}$ & Islets & Stroma & $\mathbf{I}+\mathbf{S}$ & [I + S] short \\
\hline M1 & $70.1(0-255.3)$ & $33.6(0-257.1)$ & $70.4(0-255.7)$ & $7.3(0-74.9)$ & $13.1(0-129.9)$ & $17.2(0-132.2)$ & 4.1 \\
M2 & $77.6(0-356.9)$ & $78.4(0-327.9)$ & $97.9(0-299.2)$ & $113.4(0-311.5)$ & $79.5(0-234.3)$ & $109.5(0-257.5)$ & 0.9 \\
M1/M2 & 0.9 & 0.4 & 0.7 & 0.1 & 0.2 & 0.2 & 4.5 \\
\hline
\end{tabular}

Macrophage $(\mathrm{M} \Phi)$ density is presented as median (range) of cell number per $\mathrm{mm}^{2} . \mathrm{I}+\mathrm{S}$ represents median (range) of the macrophage density in the tumor islets and stroma, which is obtained based on macrophage number per $\mathrm{mm}^{2}$ of the tumor sections and not a simple sum of the macrophage densities in the islets plus that in the stroma. $[I+S]$ long/[I+S] short represents a ratio of the median macrophage density in the tumor islets and stroma of the long survival group versus that of the short survival group. Analyzed with Mann-Whitney nonparametric test, the M1 macrophage densities in the tumor islets, stroma, and I+S of patients with long survival time are significantly higher than those of patients with short survival time $(P<0.001, P<0.05$, and $P<0.001$, respectively). The $M 2$ macrophage densities in the tumor islets, stroma, and I+S of patients with long survival time are not significantly different from those of patients with short survival time $(P=0.526, P=0.929$, and $P=0.329$, respectively). The $M 2$ macrophage density is not significantly different from the $M 1$ macrophage density in the tumor islets of patients with long survival time $(P>0.05)$. The $M 2$ macrophage density in the tumor stroma of the long survival group and the $M 2$ macrophage densities in the tumor islets or stroma of the short survival group are significantly higher than the corresponding $M 1$ macrophage densities $(P<0.01)$. 
$\left(13 / \mathrm{mm}^{2}\right)$ of the short survival group $(\mathrm{P}<0.001$ and $\mathrm{P}<0.05$, respectively) (Table 2 ). The M2 macrophage densities in the tumor islets (approximately $78 / \mathrm{mm}^{2}$ ) and stroma (approximately $78 / \mathrm{mm}^{2}$ ) of the long survival group were not significantly different from the M2 macrophage densities in the tumor islets (approximately $113 / \mathrm{mm}^{2}$ ) and stroma $\left(80 / \mathrm{mm}^{2}\right)$ of the short survival group $(\mathrm{P}>0.05)$ (Table 2). The M1/M2 ratios in the tumor islets (a ratio of 0.9 ) and stroma (a ratio of 0.4) of patients with long survival time were about 9 and 2 times of the corresponding $\mathrm{M} 1 / \mathrm{M} 2$ ratios in the tumor islets (a ratio of 0.1 ) and stroma (a ratio of 0.2) of patients with short survival time, respectively (Table 2).

\section{Correlation between the macrophage density and survival time}

Scatter plots of the macrophage density versus survival time were shown in Figure 2. The Spearman's rank correlation coefficient $\left(r_{s}\right)$ was calculated to assess any potential relationship between the macrophage density and patient's survival time. We found that the M1 macrophage densities in the tumor islets, stroma, or islets and stroma were positively associated with patient's survival time, with $\mathrm{r}_{\mathrm{s}}=0.745(\mathrm{P}<0.001)$, $0.271(\mathrm{P}<0.01)$, and $0.544(\mathrm{P}<0.001)$, respectively.
In contrast, there was no association between the M2 macrophage densities and patient's survival time $(\mathrm{P}>0.05)$.

In order to assess whether there is any value of the macrophage density in predicting prognosis, the median value of the macrophage density was used as a cut-off point to dichotomize the 100 patients into a group with a macrophage density above the median and a group with a macrophage density below the median. We found that patients with above-the-median M1 macrophage density in the tumor islets had a 1-year survival rate of $94 \%$, 3-year survival rate of $74 \%$, and 5 -year survival rate of $54 \%$, which were significantly higher than the corresponding survival rates $(42 \%, 18 \%$, and $10 \%$, respectively, $\mathrm{P}<0.001)$ in patients with below-the-median M1 macrophage density in the tumor islets (Table 3 ). Patients with above-the-median M1 macrophage density in the tumor stroma had a 1-year survival rate of $78 \%$, 3 -year survival rate of $58 \%$, and 5-year survival rate of $42 \%$, which were significantly higher than the corresponding survival rates $(58 \%, 34 \%$, and $22 \%$, respectively, $\mathrm{P}<0.01)$ in patients with below-the-median M1 macrophage density in the tumor stroma. Patients with abovethe-median M1 macrophage density in the tumor islets and stroma had a 1-year survival rate of $90 \%$, 3-year

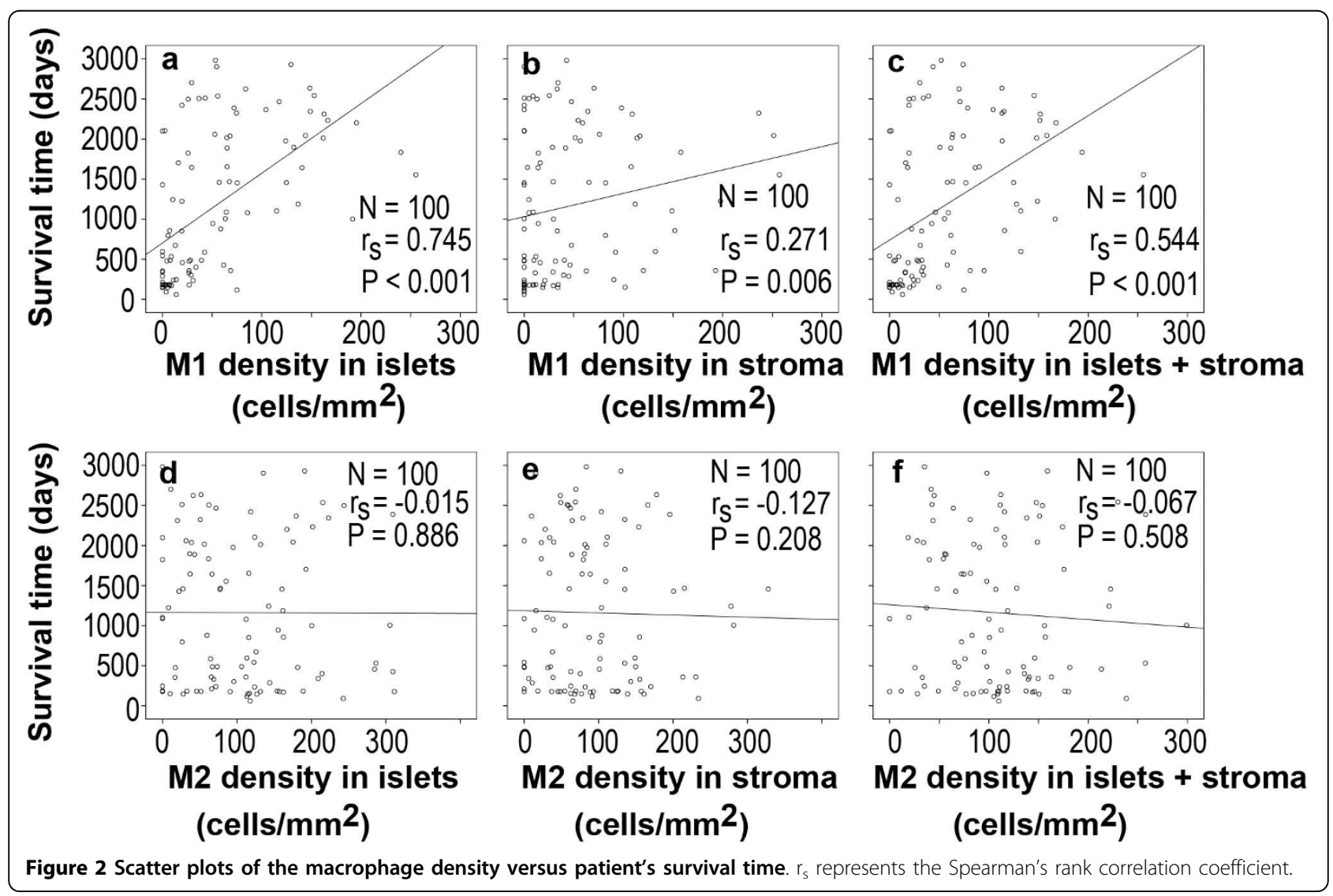


Table 3 Correlation between the density and microlocalization of macrophages and survival time in non-small cell lung cancer patients

\begin{tabular}{|c|c|c|c|c|c|c|c|}
\hline \multirow[b]{2}{*}{ Density and microlocalization } & \multirow[b]{2}{*}{$\mathbf{n}$} & \multicolumn{3}{|c|}{ Survival rate (\%) } & \multirow{2}{*}{$\begin{array}{l}P \\
\text { (univariate) }\end{array}$} & \multicolumn{2}{|l|}{ Cox regression } \\
\hline & & $\overline{1-y r}$ & $3-y r$ & $5-\mathrm{yr}$ & & Hazard ratio $(95 \% \mathrm{Cl})$ & $\begin{array}{l}\mathrm{P} \\
\text { (multivariate) }\end{array}$ \\
\hline M1 density in islets & & & & & & $\begin{array}{l}0.982 \\
(0.975-0.989)\end{array}$ & $<0.001$ \\
\hline Above median & 50 & 94 & 74 & 54 & $<0.001$ & & \\
\hline Below median & 50 & 42 & 18 & 10 & & & \\
\hline M1 density in stroma & & & & & & $\begin{array}{l}1.001 \\
(0.992-1.009)\end{array}$ & 0.889 \\
\hline Above median & 50 & 78 & 58 & 42 & 0.009 & & \\
\hline Below median & 50 & 58 & 34 & 22 & & & \\
\hline M1 density in islets + stroma & & & & & & $\begin{array}{l}1.001 \\
(0.986-1.015)\end{array}$ & 0.943 \\
\hline Above median & 50 & 90 & 70 & 50 & $<0.001$ & & \\
\hline Below median & 50 & 46 & 22 & 14 & & & \\
\hline M2 density in islets & & & & & & $\begin{array}{l}0.997 \\
(0.989-1.006)\end{array}$ & 0.535 \\
\hline Above median & 50 & 68 & 38 & 28 & 0.288 & & \\
\hline Below median & 50 & 68 & 54 & 36 & & & \\
\hline M2 density in stroma & & & & & & $\begin{array}{l}0.997 \\
(0.991-1.004)\end{array}$ & 0.430 \\
\hline Above median & 50 & 68 & 48 & 30 & 0.885 & & \\
\hline Below median & 50 & 68 & 44 & 34 & & & \\
\hline M2 density in islets + stroma & & & & & & $\begin{array}{l}1.007 \\
(0.994-1.021)\end{array}$ & 0.290 \\
\hline Above median & 50 & 64 & 42 & 30 & 0.467 & & \\
\hline Below median & 50 & 72 & 48 & 34 & & & \\
\hline
\end{tabular}

survival rate of $70 \%$, and 5 -year survival rate of $50 \%$, which were significantly higher than the corresponding survival rates $(46 \%, 22 \%$, and $14 \%$, respectively, $\mathrm{P}<$ 0.001 ) in patients with below-the-median M1 macrophage density in the tumor islets and stroma (Table 3). In contrast, the M2 macrophage densities in the tumor islets, stroma, or islets and stroma had no statistically significant association with patient's survival time. The Kaplan-Meier survival curves further illustrated association of the M1 but not M2 macrophage densities with patient's survival time (Figure 3).

In order to determine whether the macrophage density is independently associated with patient's survival time, the multivariate Cox proportional hazards analysis was used. Tumor stage, histology and grade were included in the multivariate analysis along with the macrophage density, because we found that there was no statistically significant association between the M1 macrophage density and these clinicopathological characteristics $(\mathrm{P}>0.05)$. Status of lymph node metastasis was excluded because the patients with lymph node metastasis had statistically lower M1 macrophage density in the tumor islets than the patients without lymph node metastasis $(P<0.05)$. We found that the M1 macrophage density in the tumor islets was a positive independent predictor of patient's survival time (hazard ratio $0.982,95 \% \mathrm{CI} 0.975-0.989, \mathrm{P}<0.001)$. The $\mathrm{M} 1$ macrophage densities in the tumor stroma or tumor islets and stroma, or any M2 macrophage densities had no statistically significant association with patient's survival time in the multivariate analysis (Table 3 ).

\section{Discussion}

Macrophage is a major component of inflammatory infiltrate of tumors $[11,12]$. Tumor-associated macrophages (TAMs) have complex dual functions in terms of their anti-tumor or pro-tumor effects. This functional complexity is related to the heterogeneity of macrophage population that hold a continuum of diverse functional states. At one end of the continuum are the classically activated M1 macrophages that produce effector molecules such as reactive oxygen intermediates, reactive nitrogen intermediates, and TNF $\alpha$, to limit tumor growth. At the other end are the alternatively activated M2 macrophages that promote tumor growth and metastasis by secretion of matrix-degrading enzymes, angiogenic factors and immunosuppressive cytokines/chemokines [5]. The balance of these 


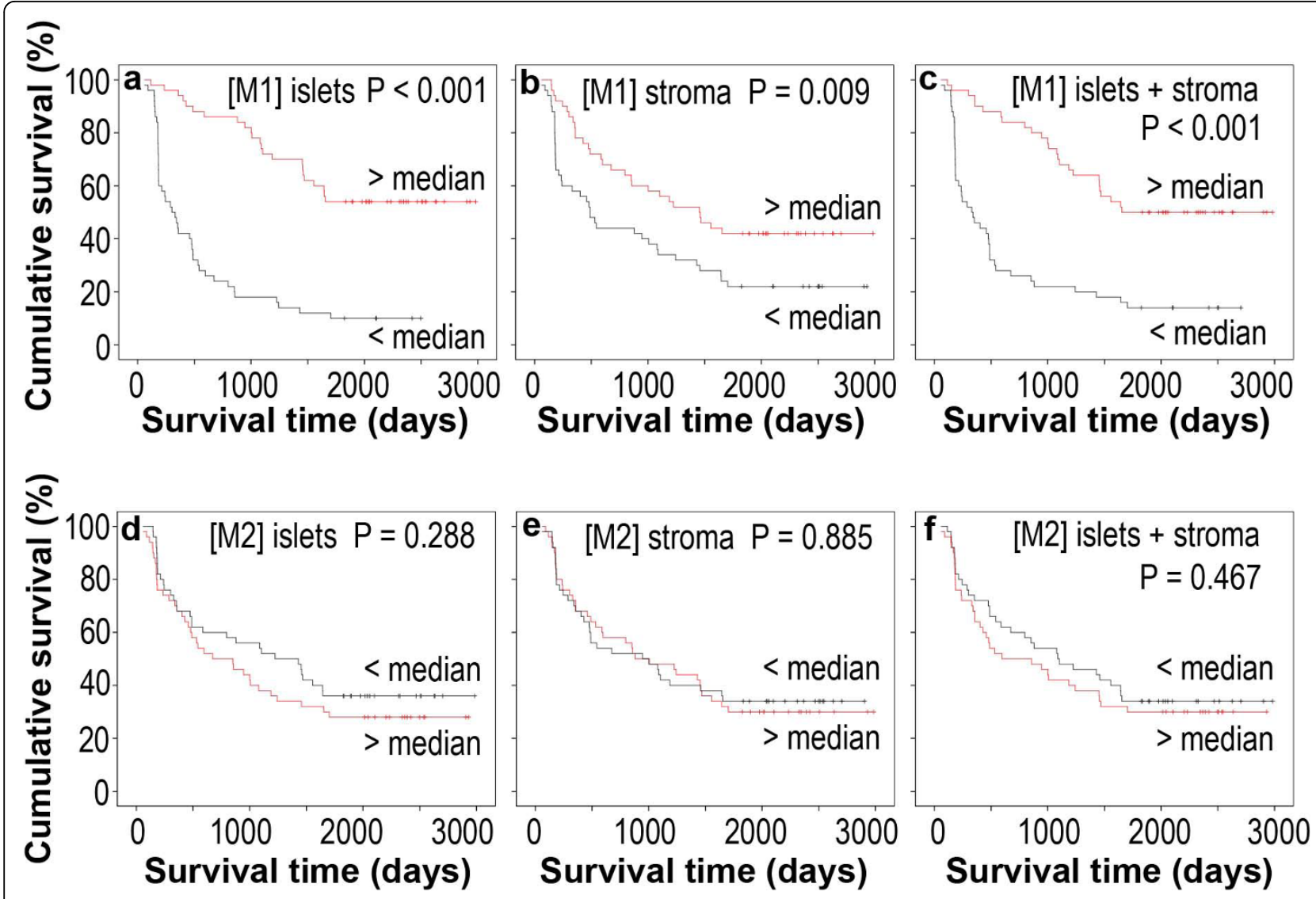

Figure 3 Kaplan-Meier survival curves. One hundred patients $(N=100)$ are divided into two groups with the macrophage densities above or below the median value. P values are obtained in comparisons of the two groups through a univariate analysis using the log-rank test. [M1] and [M2] represent the M1 macrophage density and M2 macrophage density, respectively.

macrophage forms determines the anti- or pro-tumor effects of the macrophage population [11].

Heterogeneity of macrophages may account for part of the controversies in regard to TAMs' role in prognosis. For example, Chen et al reported that TAMs are negatively associated with survival in NSCLC patients [13]. Toomey et al found that there is no association between macrophage number and prognosis of NSCLC [14]. On the other hand, Welsh et al found that the macrophage density in the tumor islets is positively associated with patient's survival [4]. Our previous study showed that the macrophage density in the tumor islets is positively associated with survival, whereas the macrophage density in the tumor stroma is negatively associated with survival [10]. We hypothesized that assessment of the M1 versus $M 2$ macrophage density in the tumor islets and stroma may provide new insights into understanding the role of TAMs in prognosis of NSCLC.

In this study, we found that in 100 cases of non-small cell lung cancer, the M2 macrophage is the predominant form of TAMs, consisting of $70 \%$ of the overall macrophage population. In comparison between the long survival and short survival groups of patients, there is no significant difference with respect to the M2 macrophage densities in the tumor islets, stroma, or islets and stroma. In contrast, the M1 macrophage densities in the tumor islets, stroma, or islets and stroma are significantly higher in the long survival group than in the short survival group. These findings imply that the M1 macrophage density may be associated with patient's survival. Indeed, using the Spearman's rank correlation coefficient analysis, we confirmed that the M1 macrophage densities in the tumor islets, stroma, or islets and stroma are positively associated with patient's survival time. Furthermore, using the median value of the M1 macrophage density as a cut-off point, patients with above-the-median M1 macrophage density in the tumor islets, stroma, or islets and stroma had significantly higher cumulative survival rates, compared to patients with below-the-median M1 macrophage density in a univariate analysis. However, in a multivariate analysis, only the M1 macrophage density in the tumor islets remains as an independent predictor of patient's survival time. These results suggest that assessment of the M1 
macrophage density in the tumor islets can be very helpful in predicting survival time of patients with NSCLC.

Our results are consistent with a recent report that the M1 macrophage density in the tumor islets is positively associated with extended survival of NSCLC patients [9]. What differs between the two studies is that we do not observe any statistically significant difference in terms of the M2 macrophage densities between the long survival and short survival groups, whereas the other study [9] found an increase of the M2 macrophage density in the tumor islets of the extended survival group. Both studies used the same clone (10D6) of mouse anti-human CD163 antigen monoclonal antibodies and similar techniques to detect the M2 macrophage. We speculate that difference in patient populations and evaluation protocols may be the main reasons for the discrepancy. Nevertheless, these studies suggest that immune responses, particularly infiltration of the M1 macrophages into the tumor islets, may play a crucial role in preventing progression of non-small cell lung cancer. The putative biological mechanisms may include direct effects of reactive oxygen/nitrogen intermediates and TNF $\alpha$ released by the M1 macrophages in close proximity to the tumor cells, and/or indirect effects through activation and recruitment of cytotoxic $\mathrm{T}$ cells [15]. It is speculated that any pharmacological interventions to induce differentiation of the M1 macrophages and/or to promote infiltration of the M1 macrophages into the tumor islets would provide therapeutic benefits to patients with non-small cell lung cancer. Future studies are needed to confirm this speculation.

The limitation of this study is that the markers used are not very specific. Although CD68 has been widely used to identify macrophages [9,16-18]], it has been found in immature CD1a-positive dendritic cells $[19,20]$. HLA-DR has been found to be expressed in the peripheral lymphocytes [21] and CD163 is also expressed in some dendritic cells [22]. Therefore, there is a possibility that some cells as identified by these three markers are not macrophages. Ideally, the cells should be stained with multiple markers. However, multiple staining would be technically challenging in handling the archived paraffin-embedded tissues.

\section{Conclusions}

This study demonstrates that the tumor-associated macrophages in non-small cell lung cancer contain two distinct forms, a CD68+/HLA-DR+ M1 form and a CD68+/CD163+ M2 form. The M1 and M2 macrophages consist of $30 \%$ and $70 \%$ of TAMs in NSCLC, respectively. The M1 macrophage densities in the tumor islets, stroma, or islets and stroma are significantly higher in patients with an average of 5-year survival, compared to patients with an average of 1-year survival. The M1 macrophage density in the tumor islets is an independent factor that can predict patient's survival time. The M2 macrophage density is not associated with patient's survival time.

\section{Acknowledgements}

The authors thank Kang Deying, Department of Evidence-Based Medicine and Clinical Epidemiology, West China Hospital, Sichuan University, for help in statistical analysis. LL is supported by grants from Science and Technology Bureau of Sichuan Province (No. 2006Z09-012, No. 2007SGY018, and No. 2009SZ0152). ZY is supported by grants from the U.S. Army Medical Research and Material Command, Department of Defense (W81XWH-05-10567), NIH/NCRR (2P20 RR020152-06), and Louisiana Cancer Research

Consortium Fund; this international collaboration is particularly supported by Tulane Framework for Global Health Seed Grant (A47599G1), NIH - Fogarty

Center - Dr. Buekens Seed Grant.

\section{Author details}

'Department of Thoracic and Cardiovascular Surgery, West China Hospital, Sichuan University, Chengdu 610041, China. ${ }^{2}$ The Third People's Hospital of Zigong City, Sichuan Province, China. ${ }^{3}$ Daping Hospital, the Third Military Medical University, Chongqing City, China. ${ }^{4}$ Departments of Structural \& Cellular Biology and Orthopaedic Surgery, Tulane Cancer Center, LCRC, Tulane Center for Aging, Tulane University School of Medicine, New Orleans, LA 70112, USA.

\section{Authors' contributions}

JM performed immunohistochemistry, evaluated the stained slides, performed statistical analysis, and drafted the manuscript. LL and GC designed and supervised the collection of data. NY collected the clinicopathological data. FD obtained the slides and evaluated the stained slides. $Z Y$ analyzed and interpreted the data and prepared the tables, figures, and manuscript text. All authors participated in manuscript preparation and approved the final version prior to submission.

\section{Competing interests}

The authors declare that they have no competing interests.

Received: 28 September 2009 Accepted: 25 March 2010

Published: 25 March 2010

\section{References}

1. Mountain CF: Revisions in the International System for Staging Lung Cancer. Chest 1997, 111(6):1710-1717.

2. Lewis CE, Pollard JW: Distinct role of macrophages in different tumor microenvironments. Cancer Res 2006, 66(2):605-612.

3. Mantovani A, Sica A, Locati M: New vistas on macrophage differentiation and activation. Eur J Immunol 2007, 37(1):14-16.

4. Welsh TJ, Green RH, Richardson D, Waller DA, O'Byrne KJ, Bradding P: Macrophage and mast-cell invasion of tumor cell islets confers a marked survival advantage in non-small-cell lung cancer. J Clin Oncol 2005, 23(35):8959-8967.

5. Mantovani A, Sica A, Locati M: Macrophage polarization comes of age. Immunity 2005, 23(4):344-346.

6. Baj-Krzyworzeka M, Szatanek R, Weglarczyk K, Baran J, Zembala M: Tumourderived microvesicles modulate biological activity of human monocytes. Immunol Lett 2007, 113(2):76-82.

7. Mantovani A, Sozzani S, Locati M, Allavena P, Sica A: Macrophage polarization: tumor-associated macrophages as a paradigm for polarized M2 mononuclear phagocytes. Trends Immunol 2002, 23(11):549-555.

8. Gordon S: Alternative activation of macrophages. Nat Rev Immunol 2003, 3(1):23-35.

9. Ohri CM, Shikotra A, Green RH, Waller DA, Bradding P: Macrophages within NSCLC tumour islets are predominantly of a cytotoxic M1 phenotype associated with extended survival. Eur Respir J 2009, 33(1):118-126. 
10. Yu N, Pu J, Pu Q, Che G, Zhang S, Liu L: Influence of Tumor Associated Macrophages Distribution on Prognosis of Non-small Cell Lung Cancer. Chin J Clin Thorac Cardiovasc Surg 2009, 16(1):44-47.

11. Mantovani A, Bottazzi B, Colotta F, Sozzani S, Ruco L: The origin and function of tumor-associated macrophages. Immunol Today 1992, 13(7):265-270.

12. Balkwill F, Mantovani A: Inflammation and cancer: back to Virchow? Lancet 2001, 357(9255):539-545.

13. Chen JJ, Yao PL, Yuan A, Hong TM, Shun CT, Kuo ML, Lee YC, Yang PC: Upregulation of tumor interleukin-8 expression by infiltrating macrophages: its correlation with tumor angiogenesis and patient survival in non-small cell lung cancer. Clin Cancer Res 2003, 9(2):729-737.

14. Toomey D, Smyth G, Condron C, Kelly J, Byrne AM, Kay E, Conroy RM, Broe P, Bouchier-Hayes D: Infiltrating immune cells, but not tumour cells, express FasL in non-small cell lung cancer: No association with prognosis identified in 3-year follow-up. Int I Cancer 2003, 103(3):408-412.

15. Ohno S, Inagawa $H$, Soma G, Nagasue $N$ : Role of tumor-associated macrophage in malignant tumors: should the location of the infiltrated macrophages be taken into account during evaluation? Anticancer Res 2002, 22(6C):4269-4275.

16. Kim DW, Min HS, Lee KH, Kim YJ, Oh DY, Jeon YK, Lee SH, Im SA, Chung DH, Kim YT, et al: High tumour islet macrophage infiltration correlates with improved patient survival but not with EGFR mutations, gene copy number or protein expression in resected non-small cell lung cancer. Br J Cancer 2008, 98(6):1118-1124.

17. Kawai O, Ishii G, Kubota K, Murata Y, Naito Y, Mizuno T, Aokage K, Saijo N, Nishiwaki Y, Gemma A, et al: Predominant infiltration of macrophages and CD8(+) T Cells in cancer nests is a significant predictor of survival in stage IV nonsmall cell lung cancer. Cancer 2008, 113(6):1387-1395.

18. Al-Shibli K, Al-Saad S, Donnem T, Persson M, Bremnes RM, Busund LT: The prognostic value of intraepithelial and stromal innate immune system cells in non-small cell lung carcinoma. Histopathology 2009, 55(3):301-312.

19. Geissmann F, Dieu-Nosjean MC, Dezutter C, Valladeau J, Kayal S, Leborgne M, Brousse N, Saeland S, Davoust J: Accumulation of immature Langerhans cells in human lymph nodes draining chronically inflamed skin. J Exp Med 2002, 196(4):417-430.

20. Caux C, Vanbervliet B, Massacrier C, Dezutter-Dambuyant C, de Saint-Vis B, Jacquet C, Yoneda K, Imamura S, Schmitt D, Banchereau J: CD34+ hematopoietic progenitors from human cord blood differentiate along two independent dendritic cell pathways in response to GM-CSF+TNF alpha. J Exp Med 1996, 184(2):695-706.

21. Nakamura H, Saji H, Aute I, Kawasaki N, Hosaka M, Ogata A, Saijo T, Kato H: Peripheral leukocytes with HLA-DR+/CD8- phenotype are associated with prognosis in patients with lung cancer. Anticancer Res 2003, 23(5b):4149-4152.

22. Maniecki MB, Moller HJ, Moestrup SK, Moller BK: CD163 positive subsets of blood dendritic cells: the scavenging macrophage receptors CD163 and CD91 are coexpressed on human dendritic cells and monocytes. Immunobiology 2006, 211(6-8):407-417.

\section{Pre-publication history}

The pre-publication history for this paper can be accessed here: http://www. biomedcentral.com/1471-2407/10/112/prepub

\section{doi:10.1186/1471-2407-10-112}

Cite this article as: Ma et al:: The M1 form of tumor-associated macrophages in non-small cell lung cancer is positively associated with survival time. BMC Cancer 2010 10:112.

\section{Submit your next manuscript to BioMed Central and take full advantage of:}

- Convenient online submission

- Thorough peer review

- No space constraints or color figure charges

- Immediate publication on acceptance

- Inclusion in PubMed, CAS, Scopus and Google Scholar

- Research which is freely available for redistribution

Submit your manuscript at www.biomedcentral.com/submit
Biomed Central 